\title{
Conservative Approach for Restoring Posterior Missing Tooth with Fiber Reinforcement Materials: Four Clinical Reports
}

Emine Sirin Karaarslan ${ }^{a}$

Ertan Ertas ${ }^{b}$

Semih Ozsevik ${ }^{c}$

Aslihan Usumez

\section{ABSTRACT}

Adhesively luted, fiber-reinforced, composite-inlay, retained fixed-partial dentures can be a clinical alternative for the replacement of missing posterior teeth in selective situations. This type of restoration allows for satisfactory esthetics and reduced tooth preparation compared to a conventional, fixed-partial denture. This clinical report describes the use of a fiber-reinforced, compositeinlay, retained fixed-partial denture as a conservative alternative for the replacement of missing posterior teeth. (Eur J Dent 2011;5:465-471)

Key words: Missing posterior teeth; Inlay; Fixed-partial denture.

a Assist. Professor, Gaziantep University, Faculty of Dentistry, Department of Restorative Dentistry, Gaziantep, Turkey.

b Assoc. Professor, Ondokuz Mayis University, Faculty of Dentistry, Department of Restorative Dentistry, Samsun, Turkey.

c DDS, PhD, Gaziantep University, Faculty of Dentistry, Department of Restorative Dentistry, Gaziantep. Turkey.

d Professor, Gaziantep University, Faculty of Dentistry, Department of Prosthodontics, Gaziantep, Turkey.

- Corresponding author: Dr. Emine Sirin Karaarslan Gaziantep University, Faculty of Dentistry,

Department of Operative Dentistry, Kampus, Gaziantep, Turkey.

Phone: +903423606060

E-mail: karaarslan.eminedgmail.com

\section{INTRODUCTION}

Various therapeutic solutions can be used to replace a single anterior and posterior missing tooth. ${ }^{1}$ The development of implant-supported restorations led to a more conservative approach to single-tooth replacement. However, some patients reject this therapeutic option either because of the higher cost or for fear of surgery. Systemic problems may also contraindicate surgery. ${ }^{2}$

Tooth-colored materials, such as all-ceramics or fiber-reinforced composites in addition to conventional metal-based systems, are recommended to withstand mastication loads, even in replacing missing posterior teeth. Ceramics or fi- 
ber-reinforced composites contribute to the positive esthetics of restoration and to avoiding discoloration of the gingiva caused by metallic systems. ${ }^{3}$ Metal-ceramic fixed-partial dentures (FPDs) have been the treatment of choice for this purpose. ${ }^{1}$ Resin-bonded FPDs with metal frameworks are considered a practical and conservative approach in dentistry, but no documentation of long-term success, especially for the replacement of posterior teeth, could be identified. The metallic framework is less than esthetically pleasing, and the metal margins of metal-ceramic FPDs may be visible, frequently making it preferable to locate the finishing line subgingivally. ${ }^{4}$ Moreover, to provide the FPDs with retention and stability, aggressive tooth reduction is necessary during preparation of abutment teeth, allowing a higher risk for pulp exposure. ${ }^{5}$

Inlay FPDs, luted via adhesive procedures, offer an alternative for the restoration of single missing teeth in posterior quadrants. The development of dentin adhesive systems has led to simpler and minimally invasive preparations. ${ }^{6}$ Although the origin of resin-bonded FPDs dates back almost 30 years, ${ }^{7}$ the prognosis and success rates for this type of prosthesis are not clear. There are various approaches to patient selection, evaluation criteria, and technical and clinical procedures. Marinello et $a^{8}{ }^{8}$ stated valuable information concerning mid-and long-term prognosis of resin-bonded FPDs.

Recently, the use of fiber-reinforced composites (FRC) has been introduced as another type of resin-bonded FPD. ${ }^{6,9}$ The most commonly used FRCs consist of glass fibers embedded in a dimethacrylate-resin matrix. ${ }^{9}$ The use of FRC in resin-bonded FPDs has been suggested because of better adhesion of the composite luting agent to the framework, good esthetics, and physiological stiffness of the framework material. ${ }^{9}$ Clinicians have a wide range of FRCs to choose from. Fabrication of reinforcing polymers is not as simple as placing a fiber into plastic. Factors affecting the durability of FRC restorations include: the properties of the fibers, matrix, and polymer; impregnation of fibers with the resin; adhesion of fibers to the matrix; the quantity of fibers; and the direction, orientation, location, construction, distribution, and position of the fibers. ${ }^{10}$ The fracture strength of materials depends on several factors, including the elastic modulus of the supporting substructure, the properties of the luting agent, the thickness of restoration, and the preparation design. ${ }^{11}$

Patient selection for an inlay FPD technique is an essential requirement for clinical success. ${ }^{6,12}$ Each situation must be evaluated to determine the location (replacement of a single posterior tooth), available room la space of $20 \mathrm{~mm}$ or less between remaining teeth ${ }^{13}$ ), and the healthy clinical condition of remaining abutments. ${ }^{14}$ Fiber-reinforced inlay FPDs are a less invasive treatment modality. ${ }^{15}$ The basic design of an FPD proximal preparation is a box-shaped preparation used to achieve optimal extension of the fiber framework and retention. ${ }^{16}$ A tub-shaped preparation may be indicated when there is insufficient space to prepare the box or to prevent irritation of the pulp of tipped teeth. ${ }^{17}$

The fiber-reinforced inlay FPD enables the original tooth anatomy to be reproduced while allowing for functionality, esthetics, and preservation of the tooth structure. Thus, when an esthetic restoration with minimal tooth reduction is desired, the fiber-reinforced inlay FPD may be a valid therapeutic option to replace a single missing tooth. ${ }^{2}$

The purpose of this clinical study was to evaluate the clinical usefulness of fiber-reinforced inlay FPD over a one-year period. This report describes the clinical treatment of patients using three types of fiber-reinforcement materials.

\section{CASE REPORTS}

The patients rejected the placement of a single-tooth implant because of the duration of therapy and requirements for surgical intervention. Likewise, conventional FPDs were refused. Thus, a fiber-reinforced inlay FPD was used for a singletooth replacement in patients refusing surgery for various psychological reasons. ${ }^{2}$ The patients selected a conservative approach to restore missing teeth because the occlusal factors were favorable labsence of bruxism, good occlusal stability, and presence of all remaining teeth).

\section{Patient 1}

A 29-year-old girl with a missing left mandibular first molar was referred to our clinic. In clinical examination, there were no problems with the soft tissues and adjacent teeth were vital (Figure 1a). 
In radiographic examination, no pathologic conditions were detected. Fiber-reinforced composite inlay FPD was indicated with the consent of the patient.

Preexisting amalgam restorations were removed and inlay preparations were done on the abutment teeth using an inlay preparation set (Inlay Preparations Set 4261; Komet, Gebr. Brasseler $\mathrm{GmbH} \&$ Co., Lemgo, Germany). The walls of the cavity were flared between $5^{\circ}$ and $15^{\circ}$. All internal line angles were rounded. ${ }^{18}$ The decision to restore the teeth with fiber-reinforced inlay FPD was primarily influenced by the size of the defect and the old restoration (Figure $1 \mathrm{~b}$ ).

After tooth preparation, retraction cords (Stayput; Roeko, Langenau, Germanyl were used to expose the subgingival finish lines. Impressions were taken using a stock tray with heavy- and light-bodied vinyl-polysiloxane (Elite H-D; Zhermack SpA., Italy). Impressions from the opposing dentition were taken with irreversible hydrocolloid (CA37; Cavex, Haarlem, Netherlands). Shade selection was done and acrylic resin provisional restorations (Temdent; Weil Dental GmbH, Rosbach, Germanyl were cemented with eugenol-free temporary cement (Cavex Temporary Cement; Cavex).

Die stone was poured, and the casts were mounted in a semiadjustable articulator. Fiber Reinforcement Material (Tescera ${ }^{\mathrm{TM}}$, Bisco, Inc. Schaumburg, IL, US) was used for fiber-reinforced composite inlay FPDs in the laboratory. Die hardener and die spacer (Tescera rubber separator, Bisco) or die separator (Tescera die separator, Biscol was applied to the model. The length of $u$ -

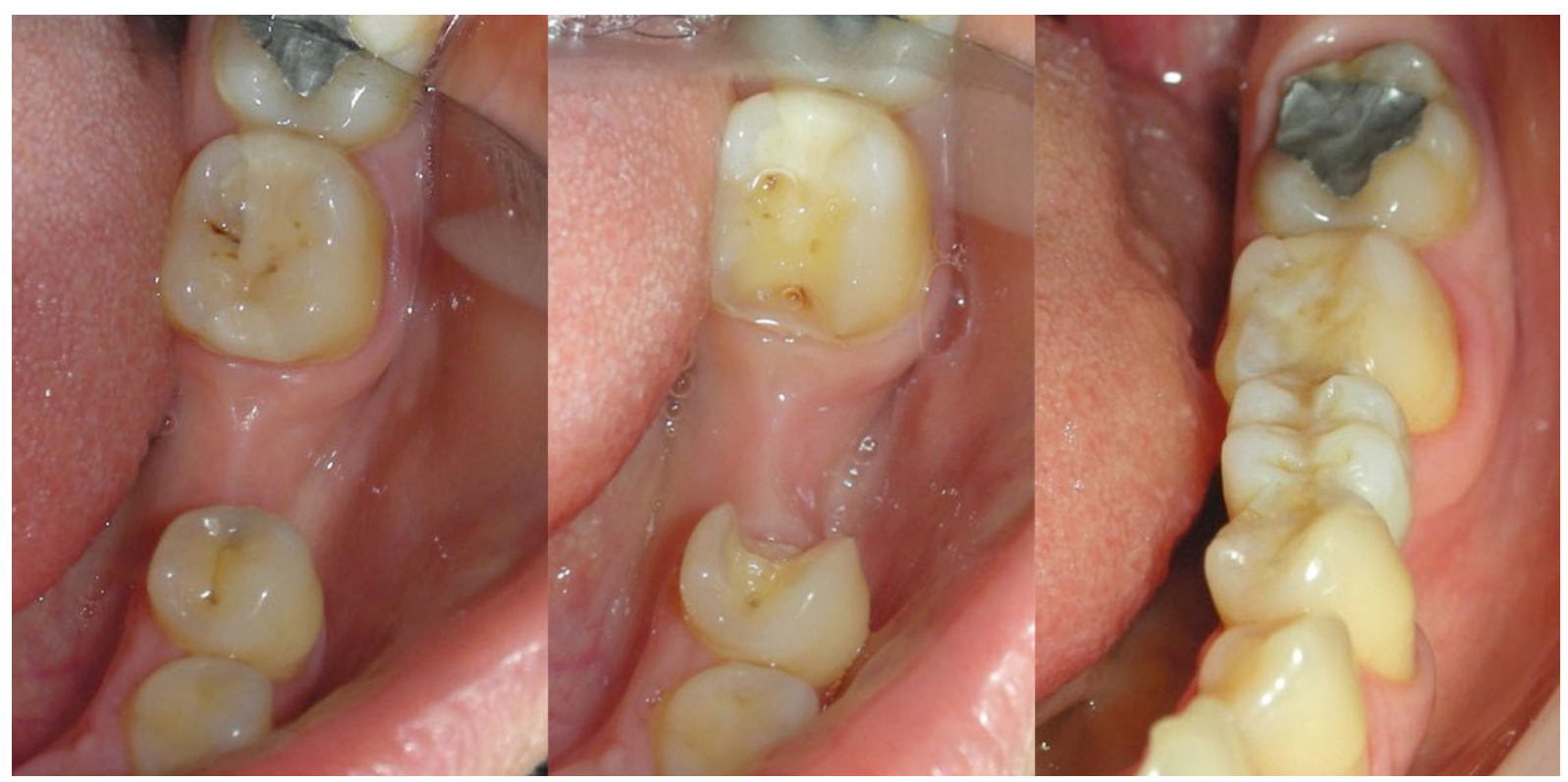

Figure 1. a. Pretreatment view of missing mandibulary left first molar. b. Preparation for class II inlays in mandibulary left first premolar and mandibulary left second molar. c. After adhesive luting, view of the fiber-reinforced composite inlay FPD.

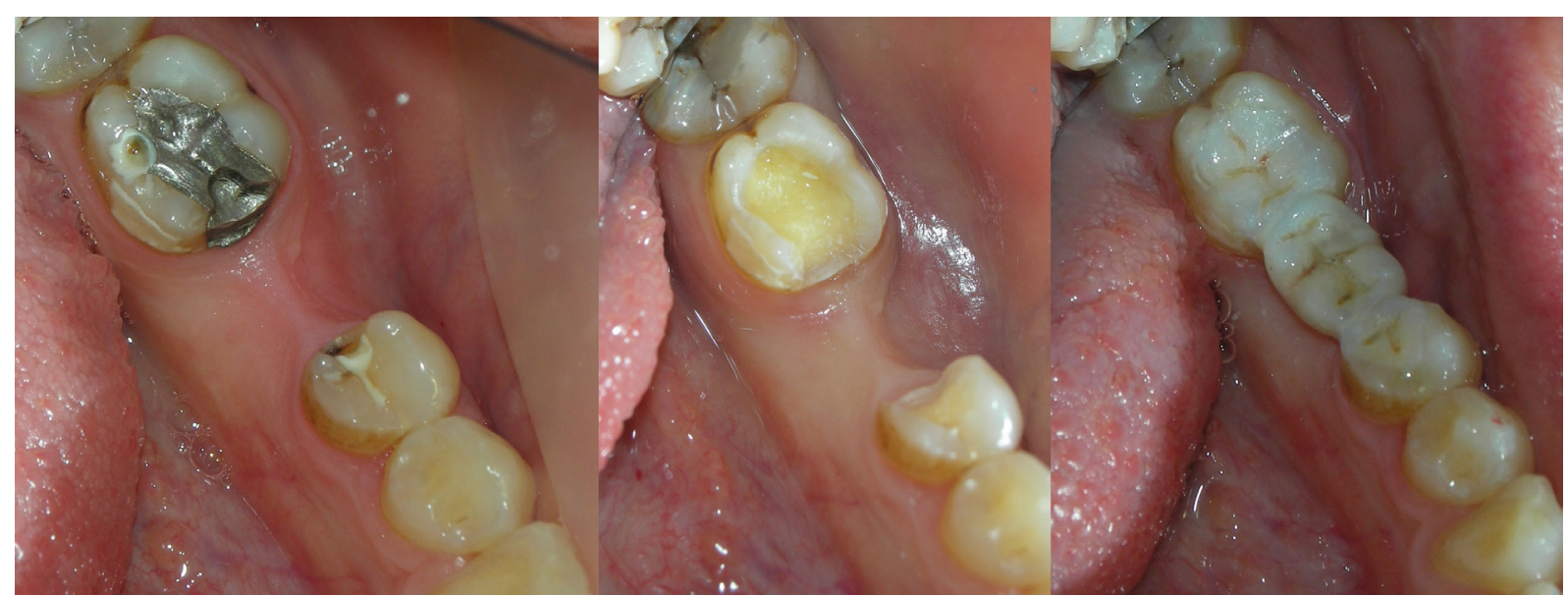

Figure 2. a. Pretreatment view of missing mandibulary left first molar. b. Preparation for class II inlays in mandibulary left first premolar and mandibulary left second molar. c. After adhesive luting, view of the fiber-reinforced composite inlay FPD. 
beam and rod were determined. A caliper was used to measure space lincluding proximal box of each abutment). Diamond bur or disc was used to cut the $u$-beam and rod to length. The minimal height requirement was $2.5 \mathrm{~mm}$. Overall preparation length was measured to determine proper length for the bundles. The bundles were cut with a pair of fine scissors. The bundles were kept as a unit and coated with flowable composite (Tesceraflo, Biscol. The flowable composite was coated in bundles using a light shield or other non-light transmitting object (cup) to protect them from light. The pre-cut u-beam and rod was cleaned by submerging in acetone. The u-beam and rod was coated with one-step or one-step plus and cured with a light-curing unit (Hilux, Ledmax-550, Benlioglu, Turkey) at $550 \mathrm{~mW} / \mathrm{cm}^{2}$ for 10 seconds. The flow- able composite was applied to the proximal box area of each abutment. The u-beam in the proximal box areas was positioned and placed into the flowable composite. The u-beam was placed with the concave surface facing the occlusal table. The u-beam was filled with flowable composite. The rod was positioned and placed into the flowable composite-filled u-beam with the aid of a brush, and excess flowable composite was removed. With a curing unit, light was applied for $20 \mathrm{sec}-$ onds. The flowable composite was applied to the preparations and the u-beam/rod assembly, and the flowable composite with coated bundles was placed on top of the assembly. The u-beam/rod/ bundle assembly was light cured for 20 seconds. After layering with composite (Tescera dentin and body, Bisco), it was placed in the light cup (Tescer-

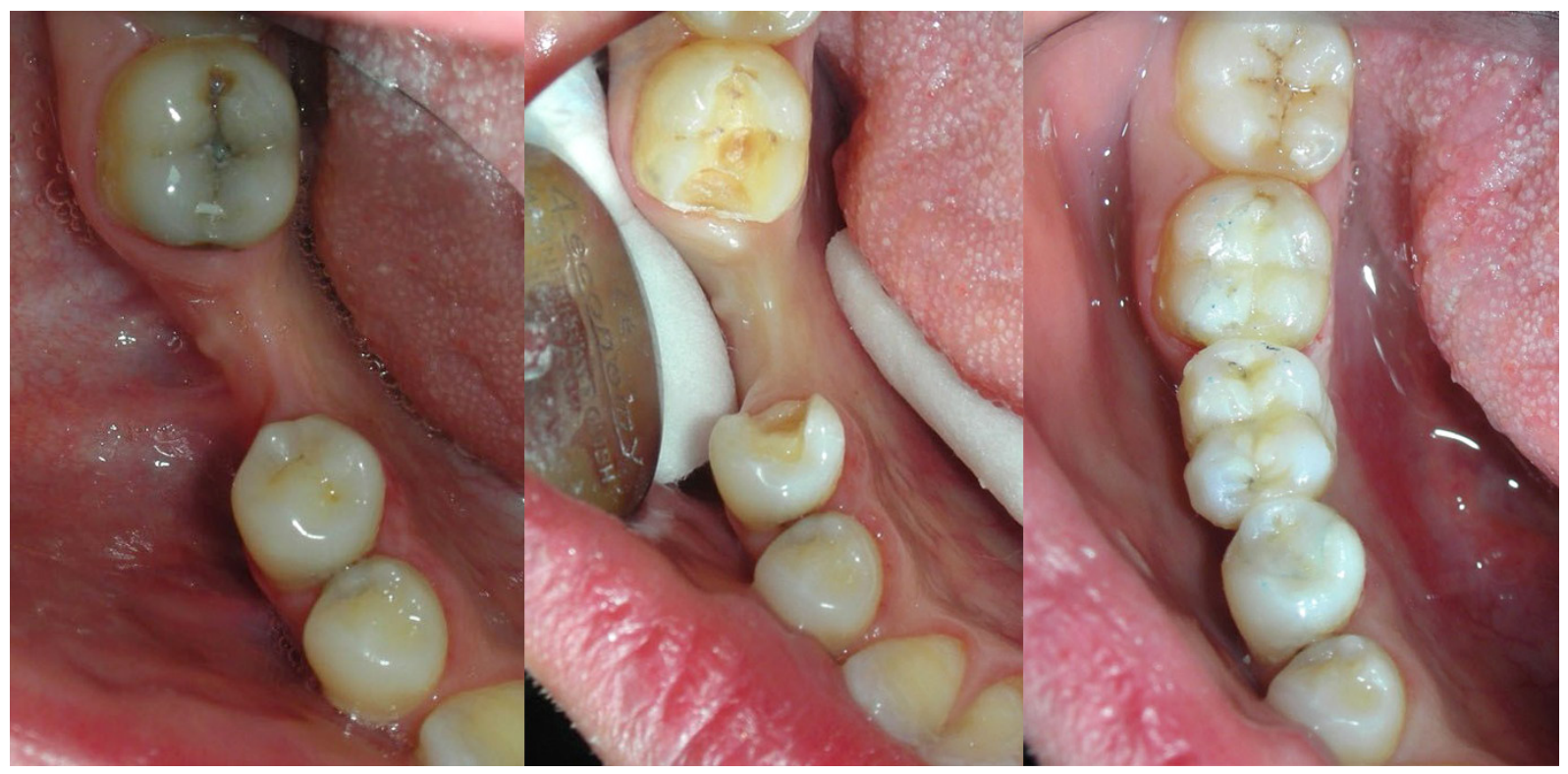

Figure 3. a. Pretreatment view of missing mandibulary right first molar b. Preparation for class II inlays in mandibulary right first premolar and mandibulary right second molar. c. After adhesive luting, view of the fiber-reinforced composite inlay FPD.
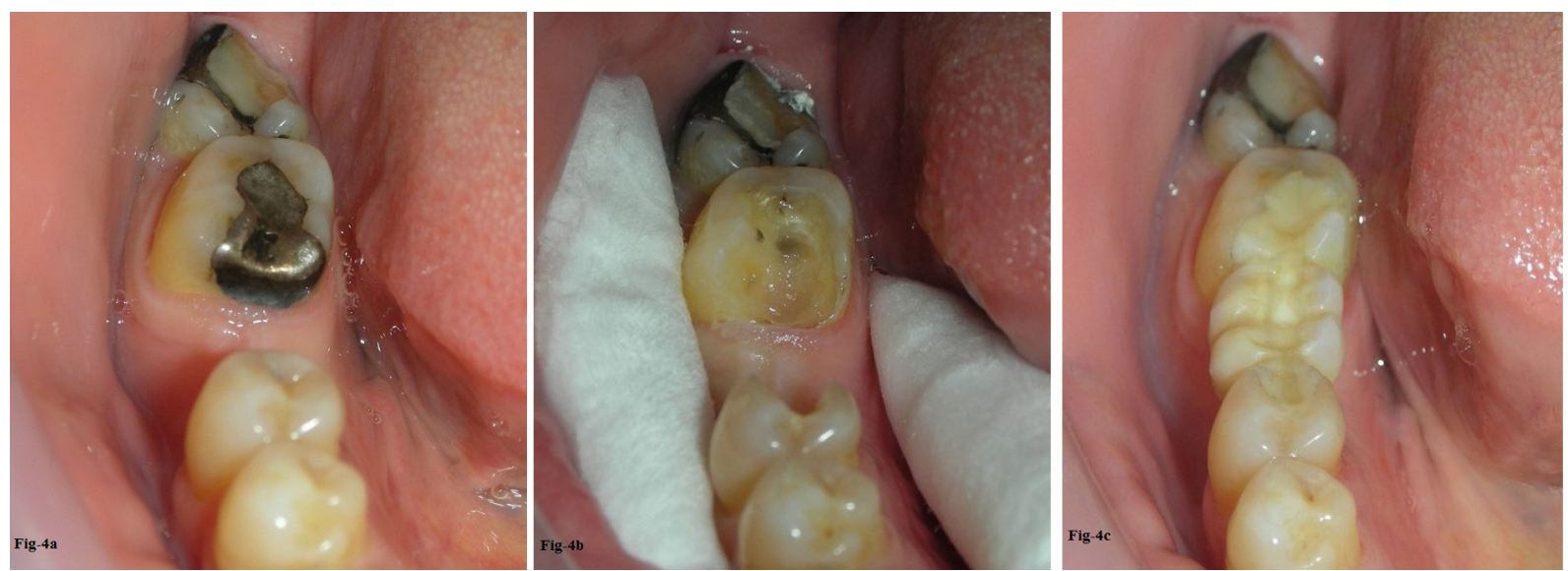

Figure 4. a. Pretreatment view of missing mandibulary right first molar b. Preparation for class II inlays in mandibulary right first premolar and mandibulary right second molar. c. After adhesive luting, view of the fiber-reinforced composite inlay FPD. 
aATL, Biscol and cured for 2 minutes under 60 PSI pressure, according to the manufacturer's instructions. It was built-up with special composite (Tescera incisal, Bisco), using the shade choice and desired sculptured contours and anatomy. The restoration was placed in the heat cup (TesceraATL, Biscol, while ensuring that restoration was covered by water, and cured for 13 minutes under $60 \mathrm{PSI}$ pressure and $130^{\circ} \mathrm{C}$ heat, according to the manufacturer's instructions. The glazing resin (Tescera, Bisco), or biscover, was applied to the restoration and light cured.

After fabrication of the restoration in the laboratory, the provisional restorations were removed; the preparations were rinsed and dried. Occlusion was evaluated with articulating paper. We evaluated the esthetics visually. To facilitate cementation, pontic inlays and cavity preparations were air-particle abraded with $50 \mu \mathrm{m}$ aluminum oxide (Microetcher II; Danville Engineering, San Ramon, CA, US). Phosphoric acid (Etch-37, Biscol was applied to cleanse the pontic inlays, which were subsequently rinsed and dried. The tooth preparations were etched with phosphoric acid etchant (Etch-37, Bisco, Schaumburg, IL, US) for 15 seconds, rinsed, and dried. A single component adhesive (One-Step Plus, Bisco) was applied to the pontic inlays and the dentin tooth structure of the cavity preparations, and the pontic inlays were bonded into place with a shaded dual-polymerized resin luting agent (Bisco), which was placed in a thin layer on the tooth preparations. The restorations were light cured for 20 seconds with a standard light-curing unit to allow the removal of the occlusal excess luting agent with an explorer or a brush. ${ }^{19}$ Contacts were evaluated and verified; the margins were finished with rotary instruments (Mani, Tochigi, Japan) and polishing discs (Sof-lex Pop On, 3M Dental Products Division, St Paul, MN, US) (Figure 1c). The glazing resin (Tescera, Bisco) was applied or biscovered to the restoration and light cured for 20 seconds with a standard light-curing unit.

Patients 2, 3 and 4

After clinical and radiographic examinations, inlay FPDs were applied to the following patients: a 32-year-old woman with a missing mandibulary left first molar, a 30-year-old man with a missing mandibulary right first molar, and a 48-year-old man with a missing mandibulary right first molar. Fiber-reinforced inlay FPDs were constructed as in the case of Patient 1 described above (Figures 2, 3 and 4).

\section{Clinical evaluation}

The restorations were rated by independent examiners with mirrors and probes using the method developed by Ryge, which is also the United States Public Health Service criteria. ${ }^{20}$ The restorations were assessed after six months and one year. Evaluation parameters included retention, color-matching ability, marginal adaption, cavosurface marginal discoloration, and postoperative sensitivity. During the clinical evaluation period, no fractures, marginal discoloration, postoperative sensitivity or secondary caries were observed in these restorations.

\section{DISCUSSION}

Minimal to no tooth preparation of the abutment teeth is desirable for the replacement of missing teeth with fiber-reinforced inlay FPD. However, depending on the clinical situation, especially in posterior applications, sufficient space is required for the fiber frame and the resin composite materials. When space is insufficient, wear of the composite may result in early failure of the restoration or in fiber exposure that may lead to plaque accumulation. ${ }^{21}$

When teeth are intact, mechanical and biological aspects must be considered in choosing the preparation design: the proximal box should be as deep as possible in the gingival direction to ensure an adequate amount of FRC and to provide maximal strength in the connection area. At the same time, the margins must be located within the enamel for better long-term marginal adaptation..$^{22}$

Song et al reported that higher fracture resistance was recorded for the box-shaped specimens. ${ }^{22}$ The box-shaped design may have provided greater surface area to resist the forces and a larger connector dimension of the fiber framework, thereby resisting the bending forces better than the tub-shaped groups in the loading tests. Theoretically, the most efficient location for fiber reinforcement is at the tensile sites, such as under the pontic. ${ }^{23}$ 
In this case report, while following the philosophy of maximum conservation of tooth structure, the box-shaped proximal preparation was performed on abutment teeth. ${ }^{24}$ The walls of the cavity were flared between $5^{\circ}$ and $15^{\circ}$. All internal line angles were rounded. ${ }^{18}$ The decision to restore the teeth with fiber-reinforced inlay FPD was primarily influenced by size of the defect and older restorations.

The mechanical properties of FRC must be improved so as to reduce the risk of clinical failure due to catastrophic fracture. ${ }^{25}$ The fracture strength of fiber-reinforced inlay FPD depends on several factors including: the preparation design, the elastic modulus of the supporting substructure, the characteristics of the manufacturing process, the occlusal load of the span, and the materials used to fabricate the prosthesis. ${ }^{2}$

In this case report Fiber Reinforcement Material (Tescera, Bisco) was used. This system consists of the following items: u-beam, rods, and bundles. The u-beam serves as the main supportive framework material for metal-free substructures. It exhibits a unique, patented design and is composed of unidirectional, pre-tensed quartz fibers bound in an epoxy resin matrix. Its $U$ shape provides the necessary strength and rigidity to prevent movement while protecting the cusps from fracture. It is available in $3 \mathrm{~mm}$ and $4 \mathrm{~mm}$ widths. The u-beam is ideal for multi-unit bridges. The rods are constructed with the same materials as the u-beam and are available in the following diameters: (1.0, 1.4, 1.8 and $2.2 \mathrm{~mm})$. When used in conjunction with the u-beam and embedded in a matrix of flowable composite (Tesceraflo), the framework achieves exceptional strength and rigidity. The bundles are strands of pre-silanated individual glass fibers. The silanation of this product allows for enhanced bonding between the resin and the glass fibers. These fibers, when coated with Tesceraflo and placed over the u-beam and rod assembly, provide additional strength to the framework resulting in superior metal-free restorations. ${ }^{26}$

\section{CONCLUSIONS}

The fiber-reinforced inlay FPD, in combination with adhesive techniques, appears to be an effective restorative solution in posterior missing tooth. However, additional evaluations still need to be carried out to reveal longer-term clinical performance of such materials.

\section{REFERENCES}

1. Kolbeck C, Rosentritt M, Behr M, Lang R, Handel G. In vitro examination of the fracture strength of 3 different fiber-reinforced composite and 1 all-ceramic posterior inlay fixed partial denture systems. J Prosthodont 2002;11:248-253.

2. Rappelli G, Coccia E. Fiber-reinforced composite fixed partial denture to restore missing posterior teeth: a case report. J Contemp Dent Pract 2005;6:168-177.

3. Rosentritt M, Behr M, Handel GJ. Fixed partial dentures:allceramics, fibre-reinforced composites and experimental systems. Oral Rehabil 2003;30:873-877.

4. Kerschbaum T, Haastert B, Marinello CP. Risk of debonding in three-unit resin-bonded fixed partial dentures. $J$ Prosthet Dent 1996;75:248-253.

5. Goodacre CJ, Bernal G, Rungcharassaeng K, Kan JY. Clinical complications in fixed prosthodontics. $J$ Prosthet Dent 2003;90:31-41.

6. Edelhoff D, Spiekermann H, Yildirim M. Metal-free inlay-retained fixed partial dentures. Quintessence Int 2001;32:269281.

7. Rochette AL. Attachment of a splint to enamel of lower anterior teeth. J Prosthet Dent 1973;30:418-423.

8. Marinello CP, Kerschbaum T, Heinenberg B, Hinz R, Peters S, Pfeiffer P, Reppel PD, Schwickerath H. First experiences with resin-bonded bridges and splints--a cross-sectional retrospective study, Part II. J Oral Rehabil 1988;15:223-235.

9. Vallittu PK, Sevelius C. Resin-bonded, glass fiber-reinforced composite fixed partial dentures: a clinical study. $J$ Prosthet Dent 2000;84:413-418.

10. Suzuki S, Nagai E, Taira Y, Minesaki Y. In vitro wear of indirect composite restoratives. J Prosthet Dent 2002;88:431436.

11. Narisawa TO. An evaluation of acoustic emission from fibre-reinforced composites. J Mater Sci 1985;20:4527-4531.

12. Olin PS, Hill EM, Donahue JL. Clinical evaluation of resin-bonded bridges: a retrospective study. Quintessence Int 1991;22:873-877.

13. Freilich MA, Duncan JP, Meiers JC, Goldberg AJ. Preimpregnated, fiber-reinforced prostheses. Part I. Basic rationale and complete-coverage and intracoronal fixed partial denture designs. Quintessence Int 1998;29:689-696.

14. Krejci I, Boretti R, Giezendanner P, Lutz F. Adhesive crowns and fixed partial dentures fabricated of ceromer/ FRC: clinical and laboratory procedures. Pract Periodontics Aesthet Dent 1998; 10: 487-498; quiz 500. 
15. Freilich MA, Karmaker AC, Burstone CJ, Goldberg AJ. Development and clinical applications of a light-polymerized fiber-reinforced composite. J Prosthet Dent 1998;80:311318.

16. Lutz F, Gohring TN. Fiber-reinforced inlay fixed partial dentures: maximum preservation of dental hard tissue. $J$ Esthet Dent 2000;12:164-171.

17. Behr M, Rosentritt M, Leibrock A, Schneider-Feyrer S, Handel G. In-vitro study of fracture strength and marginal adaptation of fibre-reinforced adhesive fixed partial inlay dentures. J Dent 1999;27:163-168.

18. Iglesia-Puig MA, Arellano-Cabornero A. Inlay fixed partial denture as a conservative approach for restoring posterior missing teeth: a clinical report. J Prosthet Dent 2003;89:443445.

19. Sorensen JA, Cruz M, Mito WT, Raffeiner O, Meredith HR, Foser HP. A clinical investigation on three-unit fixed partial dentures fabricated with a lithium disilicate glass-ceramic. Pract Periodontics Aesthet Dent 1999;11:95-106; quiz 108.

20. Ryge G, Snyder M. Evaluating the clinical quality of restorations. J Am Dent Assoc 1973;87:369-377.

21. Ozcan M, Breuklander MH, Vallittu PK. The effect of box preparation on the strength of glass fiber-reinforced composite inlay-retained fixed partial dentures. J Prosthet Dent 2005;93:337-345.

22. Song HY, Yi YJ, Cho LR, Park DY. Effects of two preparation designs and pontic distance on bending and fracture strength of fiber-reinforced composite inlay fixed partial dentures. J Prosthet Dent 2003;90:347-353.

23. Ellakwa AE, Shortall AC, Shehata MK, Marquis PM. The influence of fibre placement and position on the efficiency of reinforcement of fibre reinforced composite bridgework. $J$ Oral Rehabil 2001;28:785-791.

24. Behr M, Rosentritt M, Handel G. Fiber-reinforced composite crowns and FPDs: a clinical report. Int J Prosthodont 2003;16:239-243.

25. Kelly JR. Perspectives on strength. Dent Mater 1995;11:103110.

26. http://www.bisco.com/instructions/in134r1.pdf. 